\title{
Tapered Stent Smaller Diameter
}

National Cancer Institute

\section{Source}

National Cancer Institute. Tapered Stent Smaller Diameter. NCI Thesaurus. Code C150192.

The diameter of the portion of the tapered end of the stent that is the smaller of the two tapered ends. 